\title{
Public Description
}

National Cancer Institute

\section{Source}

National Cancer Institute. Public Description. NCI Thesaurus. Code C93636.

The textual summary of a document intended for the general population. 